\title{
The use of maternity acupuncture within a New Zealand public hospital: Integration within an outpatient clinic
}

Debra Betts $^{A}, B$ RN, BHSC, PhD • Jo McMullan ${ }^{\complement}$ RCpN, BM, DPH • Leonie Walker PhD, Dip Ac, MBAC

${ }^{\text {A Corresponding }}$

Author: debra.betts@ $\underline{\text { nzsao.com }}$

${ }^{\text {B }}$ New Zealand School of Acupuncture and Traditional Chinese Medicine

${ }^{\mathrm{C}}$ Hutt Hospital Maternity Unit

D Graduate School of Nursing and Midwifery, Victoria University

\begin{abstract}
:
Background: In June 2008, a maternity acupuncture service began operating within a Hutt Valley Hospital outpatient department, offering access to free acupuncture care for pregnancy and postnatal related conditions. This was the first and, to date, the only clinic of this type within a New Zealand hospital.

Objective: To retrospectively describe and analyse routinely collected data from 2013 and 2014, on treatment delivered in this clinic. Specifically we report on the number of women treated, their presenting condition, referral patterns and patient centred outcomes for those women presenting with back or pelvic/hip pain.

Methods: Data on the primary reason for seeking acupuncture and referral pathway were collected on a woman's initial visit. For those women presenting with back or pelvic/hip pain, a Measure Yourself Medical Outcome Profile (MYMOP) form was also completed, with a follow-up form completed on each subsequent visit until discharge.

Findings: Two hundred and sixty-one women received treatment. The majority were referred through Lead Maternity Carer (LMC) midwives and were requesting acupuncture treatment for labour preparation, back or pelvic/hip pain. MYMOP reporting demonstrated a high level of patient satisfaction, with $80 \%$ of women reporting a change in their pain that was clinically significant.

Conclusion: Data from this clinic illustrate a successful partnership between mainstream and complementary medicine, offering integrated healthcare to women in a public health system. Acupuncture offered a non-pharmaceutical treatment option and for those with back or pelvic/hip pain delivered high levels of patient satisfaction. It is hoped that this article will stimulate further interest in the under-researched area of maternity acupuncture.
\end{abstract}

Keywords: Maternity acupuncture, integrated care, complementary medicine

\section{INTRODUCTION}

The use of acupuncture for a wide range of conditions related to pregnancy is detailed in acupuncture text books (Betts, 2006; Maciocia, 1998; West, 2001). There is a growing interest from pregnant women in seeking acupuncture as a treatment (Adams, Sibbritt \& Lui, 2011; Steel et al., 2012) and a willingness from Western health practitioners to refer pregnant women (Stewart, Pallivalappila, Shetty, Pande, \& Mclay, 2014). In addition to their seeking private treatment, acupuncture is also available to women in selected United Kingdom maternity units (Yelland, 2005). Acupuncture treatment in pregnancy is provided by midwives in hospitals throughout Denmark, Finland, Germany, Norway, Sweden and Switzerland (Mårtensson, Kvist, \& Hermansson, 2009; Roemer, 2005). A recent review evaluated acupuncture during pregnancy and labour as safe when administered by a suitably qualified practitioner (Park, Youngjoo, White, \& Hyangsook, 2014).

A certificate in midwifery acupuncture has been available in New Zealand since 2007. Midwives successfully completing this qualification receive elective education points and may incorporate acupuncture into their midwifery practice, provided the woman has given informed consent (Calvert \& Pairman, 2011). It is also known that New Zealand midwives refer women to acupuncturists
(Harding \& Foureur, 2009) and that acupuncturists within New Zealand treat pregnant women for a variety of presenting complaints (Smith, Armour, \& Betts, 2014).

There is some evidence that acupuncture is beneficial in pregnancy. This includes beneficial reduction in pregnancy-related pelvic pain (Liddle \& Pennick, 2015). The largest randomised controlled trial (RCT) to date to assess the effect of acupuncture for pelvic pain involved 386 women receiving either standard treatment (pelvic belt and home exercises) alone, standard treatment plus acupuncture, or standard treatment plus physiotherapy stabilising exercises. The authors reported acupuncture as the treatment of choice for one-sided sacroiliac pain, one-sided sacroiliac pain combined with symphysis pubis pain and doubled-sided sacroiliac pain (Elden, Ladfors, Fagevik Olsen, Ostaard, \& Hagberg, 2005). Acupuncture has also been reported as beneficial for treatment of nausea in pregnancy. The largest RCT to date of 593 women reported a faster response in nausea reduction and improved quality of life for those women receiving individualised acupuncture treatment compared to a single, universally accessed acupuncture point, sham treatment, or no treatment (Smith, Crowther, \& Beilby, 2002). Beneficial effects for women diagnosed with depression during pregnancy have also been reported (Manber et al., 2010).

Acupressure and acupuncture may also help relieve labour pain 
(Smith, Collins, Crowther, \& Levett, 2011). There is no quality evidence that acupuncture improves labour outcomes when used as an induction treatment prior to a medical induction of labour (Smith, Crowther, \& Grant, 2013). However, the use of acupuncture over several weeks from 36 weeks gestation in one study reduced labour time and medical intervention (Romer, Weigel, Zieger, \& Melchart, 2000).

The use of moxibustion (providing heat over acupuncture points through a moxa stick) has been reported as beneficial over usual care for breech presentation at 34 weeks (Cardini \& Weixin, 1998) and is listed as a possible treatment in the New Zealand Evidence Based Guidelines for women with breech presentation (New Zealand Guidelines Group, 2003).

In addition, a cost effective analysis found that even if only $16 \%$ of women completed the recommended protocol involving selftreatment at home, there would be reduced hospital costs compared to usual care (van den Berg et al., 2010).

In June 2008, a maternity acupuncture service began operating within the Hutt Hospital Outpatients Department. This was initiated by the Clinical Midwifery Manager (JM) as a result of discussions between two of the authors (JM and DB) on the usefulness of acupuncture in pregnancy and the potential of a service hosted by the District Health Board (DHB) and run by the New Zealand School of Acupuncture and Traditional Chinese Medicine (NZSATCM). The partnership was set up to provide free acupuncture for women using the DHB maternity services and a clinical teaching situation for fourth year NZSATCM students. It was not intended that students would provide acupuncture in labour and birth because it is not feasible to run a clinic at the same time as attending to women in labour. Many Hutt DHB midwives, both lead maternity carer (LMC) and core, have attended the 'acupuncture for midwives' course and are encouraged to incorporate acupuncture in their practice, including during labour and birth. The treatments by NZSATCM students and midwives follow traditional acupuncture recommendations for treatment. This includes the use of moxibustion when appropriate and avoiding specific acupuncture techniques used to induce labour unless this is the aim of treatment (Betts, 2006). Women access this outpatient department directly by making an appointment through the maternity ward receptionists. Treatment rooms are provided two afternoons a week, with fourth year students from the NZSATCM providing treatment under supervision by acupuncturists registered through a professional acupuncture body and experienced in pregnancy related care. There is no charge for women attending. To the authors' knowledge, this is the first and only acupuncture clinic of this type within a New Zealand hospital.

\section{METHOD}

In 2013, the Measure Yourself Medical Outcome Profile (MYMOP) data collection was commenced to collect feedback on the women's treatment satisfaction for back or pelvic/hip pain. This was in addition to routine data collection of the number of women attending the clinic and their presenting conditions.

Information concerning the primary reason for women seeking treatment and their referral pathway to the clinic was recorded on their first visit. All women signed a NZSATCM patient consent form for treatment and data collection and an additional treatment consent form as recommended by White, Cummings, Hapwood, and MacPherson (2001).

Ethical opinion was sought from the National Ethics Advisory Committee (2012) who determined that, as the data collection for this article was part of the routine data collection procedures for the NZSATCM, formal ethical approval was not required.

\section{FINDINGS}

This clinic provides treatment for 30 weeks during the NZSATCM academic year. For 2013 this resulted in 134 women receiving treatment. For 2014 this number was 127 with the total number of treatments delivered ranging from 370 in 2013 to 408 in 2014.

\section{Referral pathways}

The main source of referral to the clinic for both 2013 and 2014, was through midwives acting as LMCs (Table 1, Figure 1). In New Zealand, midwives who are LMCs are self-employed, autonomous practitioners providing care during pregnancy, childbirth and the postpartum period. In contrast, hospital midwives employed by a DHB are called core midwives and for this data collection are grouped under the heading 'Antenatal/physio/hospital'.

Referrals from acupuncturists were from private acupuncturists for women who were unable to continue paying for private treatment. Referrals from "Yoga" came from one practitioner who ran pregnancy yoga workshops.

\begin{tabular}{|l|r|r|r|r|}
\hline Table 1. Referral to clinic 2013 and 2014 \\
\hline & $2013(n=134)$ & $2014(n=127)$ \\
\hline & $n$ & $\%$ & $n$ & $\%$ \\
\hline LMC Midwife & 98 & 73 & 80 & 63 \\
\hline $\begin{array}{l}\text { Self-referral (previous patient/ } \\
\text { friend /hospital website) }\end{array}$ & 20 & 15 & 23 & 18 \\
\hline Acupuncturist & 2 & 1 & 9 & 7 \\
\hline Antenatal /physio/hospital & 3 & 2 & 10 & 8 \\
\hline Yoga & 11 & 8 & 5 & 4 \\
\hline
\end{tabular}

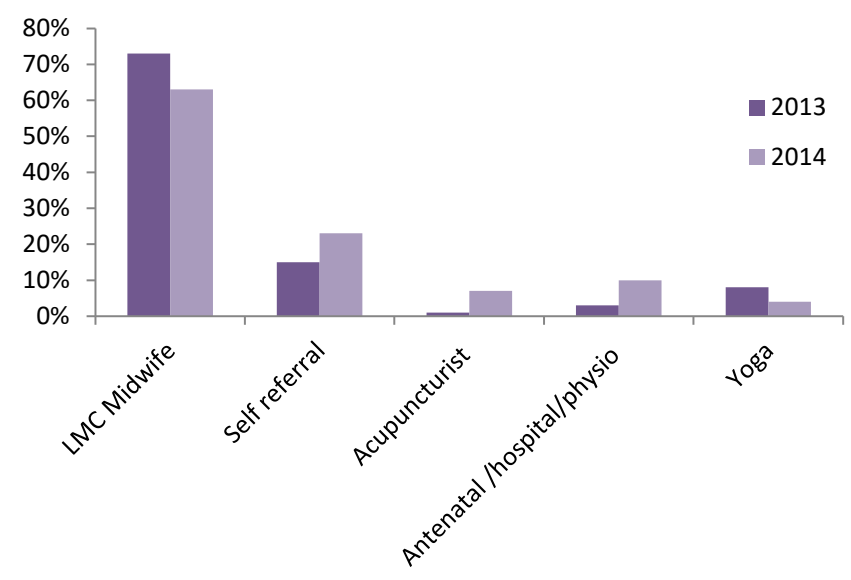

Figure 1. Referral patterns 2013 and 2014

\section{Treatment provided}

A variety of treatments were provided on the woman's initial visit (Table 2). The column labelled as "Primary" represents the presenting condition cited by the woman on her first visit as the main reason she was seeking treatment and the column labelled as "Secondary" represents those conditions also treated on this first visit following assessment.

It can be seen that the majority of women initially presented for treatment involving labour preparation (pre-birth treatment), and back or pelvic/hip pain, with 37 women (28\%) initially presenting for pre-birth in 2013 and 22 (17\%) in 2014. Thirty-four women (25\%) initially sought treatment for back pain in 2013 and 37 
women $(29 \%)$ in 2014 . For pelvic/hip pain this was 13 women $(10 \%)$ in 2013 and 19 women $(15 \%)$ in 2014. It can also be seen that there was a range of other pregnancy related conditions women received treatment for, after assessment on their first visit. This included treatment for heartburn 23 (17\%) in 2013 and 29 (23\%) in 2014 and headaches or migraines 10 (7\%) in 2013 and $10(8 \%)$ in 2014 . The heading for varicosities includes women presenting for treatment of vulval varicosities, varicose veins and haemorrhoids. Only a small number of women presented for treatment relating to induction of labour $2(1.4 \%)$ in 2013 and $3(2.3 \%)$ in 2014.

\begin{tabular}{|l|r|r|r|r|r|r|r|r|}
\hline Table 2. Treatments provided in 2013 and 2014 \\
\hline Conditions & \multicolumn{7}{|c|}{$2013 \mathrm{n}=134$} & \multicolumn{3}{|c|}{$2014 \mathrm{n}=127$} \\
\hline & Primary & Secondary & Primary & Secondary \\
\hline & $\mathrm{n}$ & $\%$ & $\mathrm{n}$ & $\%$ & $\mathrm{n}$ & $\%$ & $\mathrm{n}$ & $\%$ \\
\hline Pre-birth & 37 & 27.6 & 6 & 4.4 & 22 & 17.3 & 16 & 12.5 \\
\hline Back pain & 34 & 25.3 & 10 & 7.4 & 37 & 29.1 & 7 & 5.5 \\
\hline $\begin{array}{l}\text { Pelvic/hip } \\
\text { pain }\end{array}$ & 13 & 9.7 & 13 & 9.7 & 19 & 14.9 & 4 & 3.1 \\
\hline Other & 19 & 14.1 & 11 & 8.5 & 11 & 8.6 & 0 & 0 \\
\hline Nausea & 13 & 9.7 & 2 & 1.4 & 11 & 8.6 & 0 & 0 \\
\hline Varicosities & 5 & 3.7 & 5 & 3.7 & 4 & 3.1 & 7 & 5.5 \\
\hline Emotional & 5 & 3.7 & 4 & 2.9 & 4 & 3.1 & 10 & 7.8 \\
\hline Breech & 4 & 2.9 & 0 & 0 & 2 & 1.5 & 0 & 0 \\
\hline Induction & 2 & 1.4 & 0 & 0 & 3 & 2.3 & 0 & 0 \\
\hline Insomnia & 2 & 1.4 & 6 & 4.4 & 4 & 3.1 & 0 & 0 \\
\hline $\begin{array}{l}\text { Headache/ } \\
\text { migraine }\end{array}$ & 0 & 0 & 10 & 7.1 & 4 & 3.1 & 10 & 7.8 \\
\hline Heartburn & 0 & 0 & 23 & 17.1 & 0 & 0 & 29 & 22.8 \\
\hline Postnatal & 0 & 0 & 0 & 0 & 6 & 4.7 & 0 & 0 \\
\hline
\end{tabular}

The heading of "Other" included a variety of presentations where women mentioned they also were experiencing, or had been diagnosed with a pregnancy related condition such as: rib pain, upper back pain, wrist pain, carpal tunnel syndrome, uterine pain, gestational diabetes, hypertensive disorders, bleeding in early pregnancy, requiring early pregnancy support, oedema, cholecystitis, pregnancy rashes, eczema, thrush, sinus pain, chest infections and diarrhoea.

\section{Data collection and results for MYMOP}

For women presenting with back or pelvic/hip pain a MYMOP form was completed. MYMOP is a patient centred questionnaire that asks the patient to describe their problem in their own words and then rate it on a scale from 0 to 6 . This is a scale with 0 being "as good as it could be" and 6 being "as bad as it could be." The same scale of 0-6 is used for any associated symptom, an associated activity they find difficult and a general wellbeing scale.

The questionnaire was completed during the first visit and then repeated on all subsequent visits. A mean change was calculated from the initial and final score to obtain a profile score. This represents a score that is clinically significant rather than statistically significant; with a reduced change above one representing a positive change seen as important to the patient receiving the treatment. MYMOP is an outcome measure that can be used to collect clinically significant scores from individuals seeking treatment (Bovery et al., 2005). This problem-specific questionnaire has been shown to be a reliable tool when used in acupuncture studies to measure the quality of changes considered important for the individuals receiving treatment (Hull, Page, Skinner, Linville, \& Coeytaux, 2006; Paterson, 1996; Paterson \& Britten, 2003; Paterson, Unwin, \& Joire, 2010).

Although a total of 107 women presented with a condition suitable for MYMOP collection, there was a high number of incomplete and incorrect forms. This group included forms that were not initiated or mistakes due to student inexperience with data collection, such as circling two numbers on the scale, or changing symptoms during the treatment weeks. In addition, there were women who only received one treatment and were therefore unable to complete their MYMOP form. Due to the high number of incomplete forms in 2013, students in 2014 received additional training and their data collection was more closely monitored which resulted in improved data collection (Table 3).

\begin{tabular}{|c|c|c|c|c|}
\hline & \multicolumn{2}{|c|}{2013} & \multicolumn{2}{|c|}{2014} \\
\hline & $n$ & $\%$ & $n$ & $\%$ \\
\hline $\begin{array}{l}\text { Total presentations suitable for } \\
\text { MYMOP }\end{array}$ & 51 & & 56 & \\
\hline Forms not initiated or incomplete & 23 & 45 & 8 & 14 \\
\hline $\begin{array}{l}\text { Only one visit - therefore MYMOP } \\
\text { could not be completed }\end{array}$ & 12 & 24 & 19 & 34 \\
\hline Forms completed & 16 & 31 & 29 & 52 \\
\hline
\end{tabular}

\section{MYMOP profile scores}

Of the 45 completed MYMOP forms, 36 (80\%) had a profile score that reduced by more than one point, which can be seen as clinically significant - that is, representing a change that is of importance to those women. Five women (11\%) had a score below 0.5 , i.e., an improvement which can be seen as not clinically significant, with four women (9\%) showing minimal improvement from the women's perspective (Figure 2).

Figure 2. Percentages of women over the range of possible

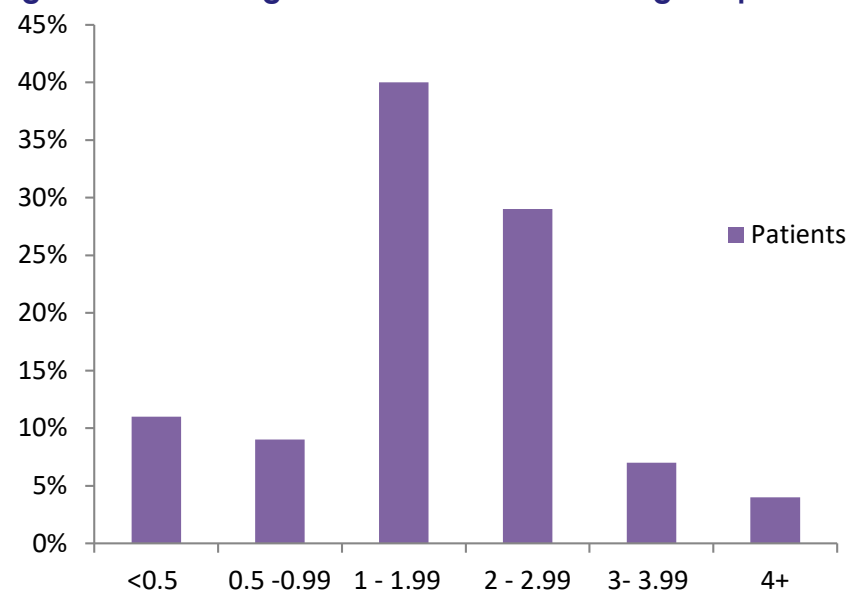

MYMOP profile scores, for 2013 and $2014(n=45)$

\section{DISCUSSION}

The majority of women seeking treatment through this maternity acupuncture service were referred by LMCs. The treatments most commonly sought by women at their initial visit were for labour preparation, back pain, or pelvic/hip pain. It was also apparent that, although women initially sought treatment for these specific conditions, following assessment they also received treatment for a wider range of problems such as heartburn, insomnia, emotional considerations, varicosities, headaches and migraines. 
The literature on acupuncture use in hospitals focuses on acupuncture or acupressure to induce, or use as pain relief during, labour, although there have also been reports of acupuncture use within an outpatients' department. A small exploratory study, reporting on 37 women receiving acupuncture through an Australian clinic, found that the majority of women became aware of the service through midwives and initially presented for back pain, pelvic pain and hip pain (Hope-Allen, 2004). In addition, an audit of a midwifery acupuncture service in the United Kingdom specifically for women with back or pelvic/hip and rib pain reported on 43 women receiving treatment (de Jonge-Vors, 2011). Although only $35 \%$ demonstrated measurable pain reduction on a pain scale, $85 \%$ of the 21 women surveyed reported an improvement in their symptoms.

A larger retrospective study did report on 5885 women receiving acupuncture treatment at a German hospital (Romer, Zieger, \& Melchert, 2013). However, although the authors reported that 43 women presented with an intrauterine death, there was no further reporting on the number of women receiving treatment for specific pregnancy-related conditions. It therefore remains unknown what conditions the majority of women were being treated for. The authors also claim their findings demonstrate that all acupuncture points, including those used to induce labour, are safe at all times in pregnancy. However, they give no details of specific points used. The majority of points they list as "forbidden" are not used to induce labour and therefore are not regarded as a relevant concern by other practitioners (Betts, 2006; Betts \& Budd, 2011). In addition, their list of "forbidden points" contain points now recommended as appropriate for labour preparation (Betts, 2006; Roemer, 2005; Romer et al., 2000). The authors of this article agree there are specific acupuncture points, traditionally considered forbidden due to the potential to induce labour or miscarriage, that now are considered as useful to prepare women for labour from 36 weeks. However, the failure of the authors to report if these labour preparation points were used to treat women before 36 weeks conveys no confidence to their claim that these labour preparation points can be used at any time in pregnancy without caution.

Back and pelvic pain are common conditions in pregnancy where pain usually increases as the pregnancy advances, interfering with a woman's ability to work, her daily activities, and quality of sleep (Mogren, 2006). In randomised controlled trials, the use of acupuncture in the treatment of back and pelvic pain has been demonstrated to be beneficial (Pennick \& Liddle, 2013).

Although the use of MYMOP to determine a profile score only reveals the woman's perception of any improvement, it does provide the opportunity to assess how women perceive acupuncture within a clinical care environment. Unlike controlled trials there were no entry criteria with midwives able to refer and women able to selfrefer at any time during their pregnancy, and the treatment was provided to women presenting with chronic back pain conditions, as well as acute back and pelvic pain. While in New Zealand there is an accident compensation system intended to provide free and low cost treatment options for any back pain relating to an injury, there were incidences of women presenting with chronic back pain who had not received any medical assessment or treatment at the time of a previous accidental injury. Where possible, referral was initiated to address this; however, the cost for seeking further medical assessment was voiced as a barrier by many women.

Pre-birth acupuncture was also sought by women. This involves using a weekly acupuncture treatment from 36 weeks gestation to prepare a woman for labour. The aim of treatment is to aid cervical ripening, promote a cephalic presentation and anterior position for the baby, and address any presenting emotional and/ or physical pain issues for the women. There is limited research on the use of pre-birth acupuncture. However, a German randomised controlled trial involving 1000 primiparous women demonstrated that the use of specific acupuncture points, delivered once a week from 36 weeks, positively influenced Bishop scores, resulting in a shorter duration of labour and fewer caesarean sections (Romer et al., 2000). In addition, an observation study in Wellington, New Zealand, involving 14 LMC midwives reported that for 169 first-time mothers and multiparas, pre-birth acupuncture resulted in fewer medical inductions and fewer caesarean sections compared to those women receiving usual care (Betts \& Lennox, 2006). Although further randomised trials are required to further establish the efficacy of pre-birth treatment, it was evident that in our clinic women sought out acupuncture as an option for their labour preparation.

\section{STRENGTHS AND LIMITATIONS}

To the authors' knowledge, this is the first paper reporting in detail on a large number of women receiving maternity acupuncture through a public hospital outpatient service. Due to the limited nature of these findings, caution with interpretation or generalisation is required. As there was no control group to compare findings for the MYMOP scores, these scores only reflect the women's personal satisfaction and it is possible that these scores were influenced by confounding factors, such as time to rest, attention paid, and desire to please the acupuncturist. It was a limitation that a MYMOP form was not initiated or completed correctly for all women presenting with back or pelvic/hip pain. It was also a limitation that not all women who initiated a MYMOP form returned for further treatment. It was a practical consideration that, due to limited clinic hours available for appointments, women receiving their first treatment did not always receive a follow-up appointment. Changes have now been made to the booking system so that all women receive three appointments on booking to ensure that follow-up appointments are available in a relevant time frame. The preliminary results offered in this paper identify potential issues for future investigation. At the time of writing we can confirm that this clinic continues to operate as a free outpatient service at Hutt Valley Hospital. Data collection continues with demographic data now being collected for future reporting. Changes have also been implemented to improve the completion of MYMOP forms and data collection has commenced for adverse events reporting. We are also currently planning to further explore and report on women's experience of their treatment through this clinic.

\section{CONCLUSION}

The creation of this maternity acupuncture service arose from openness on the part of the Hutt Valley maternity service to explore positive feedback it had received concerning acupuncture, and an acupuncture school seeking clinical placement within a hospital for its students. This clinic illustrates a successful partnership between mainstream and complementary medicine, offering research opportunities and integrated healthcare to a large number of women in a public health system.

The use of acupuncture in this context provides these women with a non-pharmaceutical treatment option. As we have outlined above, further data collection is underway for this clinic. It is the authors' hope that this article will stimulate further interest in this under-researched area of practice. 


\section{ACKNOWLEDGEMENTS AND CONFLICT OF INTEREST STATEMENT}

Debra Betts is employed as the clinical supervisor at Hutt Hospital Maternity Acupuncture Clinic by the New Zealand School of Acupuncture and Traditional Chinese Medicine. The other two authors report no conflict of interest.

\section{REFERENCES}

Adams, J., Sibbritt, D., \& Lui, C. (2011).The use of complementary and alternative medicine during pregnancy: A longitudinal study of Australian women. Birth, 38, 200-6.

Betts, D. (2006). The essential guide to acupuncture in pregnancy \& childbirth. Hove, England: The Journal of Chinese Medicine Ltd.

Betts, D., \& Budd, S. (2011). 'Forbidden points' in pregnancy: historical wisdom? Acupuncture in Medicine, 29(2), 137-9.

Betts, D., \& Lennox, S. (2006). Acupuncture for pre-birth treatment: An observational study of its use in Midwifery practice. Medical Acupuncture, 17(3), 16-19.

Bovery, M., Horner, C., Shaw, J., Linthwaite, P., Mole, P., Pedley, B., \& Robinson, N. (2005). Engaging in the audit of acupuncture practice. Journal of Alternative \& Complementary Medicine, 11(2), 293-298.

Calvert, S., \& Pairman, S. (2011). Midwifery Scope of Practice on acupuncture and frenetomy. Midwifery News, June, 41-43.

Cardini, F., \& Weixin, H. (1998). Moxibustion for correction of breech presentation. The Journal of the American Medical Association, 280, 15801584.

de Jonge-Vors, C. (2011). Reducing the pain: Midwifery acupuncture service audit in Birmingham. The Practising Midwife, November, 1-4.

Elden, H., Ladfors, L., Fagevik Olsen, M., Ostaard, H., \& Hagberg, H. (2005). Effects of acupuncture and stabilising exercises as adjunct to standard treatment in pregnant women with pelvic girdle pain: Randomised single blind controlled trial. British Medical Journal, 330, doi: http://dx.doi.org/10.1136/bmj.38397.507014.E0

Harding, D., \& Foureur, M. (2009). New Zealand and Canadian midwives' use of complementary and alternative medicine. New Zealand College of Midwives Journal, 40, 7-12.

Hope-Allen, N. (2004). The use of acupuncture in maternity care: a pilot study evaluating the acupuncture service in an Australian hospital antenatal clinic. Complementary Therapies in Nursing \& Midwifery, 10, 229-32

Hull, S. K., Page, C. P., Skinner, B. D., Linville, J. C., \& Coeytaux, R. R. (2006). Exploring outcomes associated with acupuncture. Journal of Alternative \& Complementary Medicine, 12, 247-254.

Liddle, S.D., \& Pennick, V. (2015). Interventions for preventing and treating low-back and pelvic pain during pregnancy. Cochrane Database of Systematic Reviews, 9. CD001139.doi: 10.1002/14651858.CD001139.

Maciocia, G. (1998). Obstetrics and Gynecology in Chinese Medicine. New York: Churchill Livingstone.

Manber, R., Schnyer, R., Lyell, D., Chambers, A., Caughey, A., Druzin, M., \& ... Allen, J. (2010). Acupuncture for depression during pregnancy: a randomized controlled trial. Obstetrics and Gynecology, 115(3), 511520. doi:10.1097/AOG.0b013e3181cc0816

Mårtensson, L., Kvist, L. J., \& Hermansson, E. (2009). A national survey of how acupuncture is currently used in midwifery care at Swedish maternity units. Midwifery 27(1), 87-92.

Mogren, I. M. (2006). Perceived health, sick leave, psychosocial situation, and sexual life in women with low-back and pelvic pain during pregnancy. Acta Obstetricia et Gynecologica Scandinavica, 85, 647-56.

National Ethics Advisory Committee. (2012). Ethical Guidelines for Observational Studies: Observational research, audits and related activities. Wellington: Ministry of Health. Retrieved from http://neac.health.govt. $\mathrm{nz} /$ streamlined-ethical-guidelines-health-and-disability-research

New Zealand Guidelines Group. (2003). Care of women with breech presentation or previous caesarean birth. Wellington: New Zealand Guidelines Group. Retrieved from http://www.health.govt.nz/ publication/care-women-breech-presentation-or-previous-caesarean-birth Park, J., Youngjoo, S., White, A. R., \& Hyangsook, L. (2014). The safety of acupuncture during pregnancy: A systematic review. Acupuncture in Medicine, 32(3), 257-266. doi:10.1136/ acupmed-2013-010480
Paterson, C. (1996). Measuring outcomes in primary care: a patient generated measure, MYMOP, compared with the SF-36 health survey. The BMJ, 312, 1016-1020.

Paterson, C., \& Britten, N. (2003). Acupuncture for people with chronic illness: combining qualitative and quantitative outcome assessment. Journal of Alternative \& Complementary Medicine, 9, 671-681.

Paterson, C., Unwin, J., \& Joire, D. (2010). Outcomes of traditional Chinese medicine (traditional acupuncture) treatment for people with long-term conditions. Complementary Therapies in Clinical Practice, 16, 3-9.

Pennick, V., \& Liddle S. D. (2013). Interventions for preventing and treating pelvic and back pain in pregnancy. Cochrane Database of Systematic Reviews, 8. CD001139.doi: 10.1002/14651858

Roemer, A. (2005). Medical acupuncture in pregnancy. Stuttgart: Thieme.

Romer, A., Weigel, M., Zieger, W., \& Melchart, F. (2000). Prenatal acupuncture: effects on cervical maturation and duration of labour. Geburtshilfe und Frauenheilkunde, 6(10), 513-8.

Romer, A., Zieger, W., \& Melchert, F. (2013). Prohibition of acupuncture points during pregnancy - an outdated tradition or objective evidence? Deutsche Zeitschrift Für Akupunktur, 56,10-13.

Smith, C. A., Armour, M., \& Betts, D. (2014). Treatment of women's reproductive health conditions by Australian and New Zealand Acupuncturists. Complementary Therapies in Medicine, 22(4), 710-18.

Smith, C.A., Collins, C.T., Crowther, C.A., \& Levett, K. M. (2011). Acupuncture or acupressure for pain management in labour. Cochrane Database of Systematic Reviews, 7. CD009232. doi: 10.1002/14651858.

Smith, C., Crowther, C., \& Beilby, J. (2002). Acupuncture to treat nausea and vomiting in early pregnancy: A randomized controlled trial. Birth, 29(1), 1-9.

Smith, C.A., Crowther, C.A., \& Grant, S.J. (2013). Acupuncture for induction of labour. Cochrane Database of Systematic Reviews, 8. CD002962. doi: 10.1002/14651858.CD002962.

Steel, A., Adams, J., Sibbritt, D., Broom, A., Gallois, C., \& Frawley, J. (2012). Utilisation of complementary and alternative (CAM) practitioners within maternity care provision: Results from a nationally representative cohort study of 1,835 pregnant women. BMC Pregnancy and Childbirth, 12, 146. doi:10.1186/1471-2393-12-146

Stewart, D., Pallivalappila, A.R., Shetty, A., Pande, A., \& Mclay, J. S. (2014). Healthcare professional views and experiences of complementary and alternative therapies in obstetric practice in North East Scotland: A prospective questionnaire survey. BJOG, 121, 1015-9.

van den Berg, I., Kaandorp, G. C., Bosch, J. L., Duvekot, J. J., Arends, L. R., \& Hunink, M. G. (2010). Cost-effectiveness of breech version by acupuncture-type interventions on BL 67, including moxibustion, for women with a breech foetus at 33 weeks gestation: A modelling approach. Complementary Therapies in Medicine, 18(2), 67-77. doi: 10.1016/j.ctim.2010.01.003

West, Z. (2001). Acupuncture in pregnancy and childbirth. Edinburgh: Churchill Livingstone.

White, A., Cummings, M., Hapwood, V., \& MacPherson, H. (2001). Informed Consent for Acupuncture - An information leaflet developed by consensus. Acupuncture in Medicine 19(2), 123-129.

Yelland, S. (2005). Acupuncture in midwifery. Edinburgh: Elsevier.

\section{Accepted for publication August 2016}

Betts, D., McMullan, J. \& Walker, L. (2016). The use of maternity acupuncture within a New Zealand public hospital: Integration within an outpatient clinic. New Zealand College of Midwives Journal, 52, 45-49.

http://dx.doi.org/10.12784/nzcomjnl52.2016.7.45-49 